\title{
Clinical Staging of COPD
}

\author{
Gilbert Berdine MD
}

The traditional means to divide COPD patients into groups or stages are based on the FEV1. This does make sense as nobody would question that - all other things being equal - a person with a worse FEV1 is worse off than a person with a better FEV1. The problem is that all other things are never equal. FEV1 is an essential element in making a diagnosis of COPD. Serial determinations of FEV1 are very helpful in detecting important changes of condition in individuals. Yet, despite the value of FEV1, it is only a rough predictor of clinical course.

In my own practice, I stage patients with COPD based on their response to steroids when treated for exacerbations. This grouping is helpful in predicting how long patients will be in the hospital and what kinds of steroid tapers might work without relapse. I find considerable overlap of FEV1 with these stages.

I left academia in 1989 to enter private practice. I returned to academia in 2009 when my health no longer permitted my private practice workload. The following staging strategy is based on 20 years of following patients with COPD longitudinally within a private practice based in both clinic and hospital settings. I use conventional treatment strategies for patients with various combinations of albuterol, anti-cholinergic agents, long acting beta agonists and inhaled steroids. I also use conventional criteria for treatment with supplemental oxygen and pulmonary rehabilitation.

Stage I is early disease. My standard outpatient treatment of exacerbations is triamcinolone $80 \mathrm{mg}, \mathrm{IM} \times 1$ and prednisone $40 \mathrm{mg}$, PO QDaily for 4 days. The results are nearly always dramatic. The patients frequently claim they have not felt this good in years, and they want 'the shot' for their next exacerbation. The FEV1 improves dramatically and the patients claim no symptoms. This benefit usually lasts many months. For the unusual patient in Stage I who ends up in the hospital, a few doses of IV meth- ylprednisolone $125 \mathrm{mg}$, IV will result in dramatic improvement. These patients generally can go home in a day or two with a short term auto-pilot steroid taper.

The patients in Stage I are warned about the side effects of steroids. Part of the education is that if shots can be spaced out at least 3 months apart, there will be few, if any, cumulative side effects such as facial swelling or easy bruising. This sets up the transition into Stage II. As the Stage I patient gets worse, the time between exacerbations becomes shorter.

Stage II patients do not require follow up visits. They will return 3 months to the day from their last steroid shot. They still get complete relief from the therapy, but it will not last the necessary 3 months to avoid problems. They endure some difficulty to avoid taking daily steroids. Hospitalizations are still uncommon, but Stage II hospitalizations take longer and there may be a relapse near the end of auto-pilot steroid taper.

Stage III patients can no longer wait 3 months for a shot. I generally start these patients on daily steroids. They no longer get completely well after each exacerbation, but they still get considerable relief with bursts of high dose steroids. Hospitalizations become common. The patient may take 2-3 days of very high doses of parenteral steroids to improve. Length of stay is longer. Attempts to auto-pilot steroid taper usually fail at the lower doses. These patients require individualization of steroid dosing and taper with frequent ups and downs on the dose. A good result is if the patient does well on $10 \mathrm{mg}$ prednisone per day or less. Steroid side effects such as skin blotching, moon facies and fluid retention are common.

In Stage IV, the patient is never well. Even immediately after high doses of steroids, the patient has considerable symptoms. Partial relief is the best that can be achieved. Hospitalizations are frustrating for doctor and patient alike, because little or no benefit is 
perceived with high doses of parenteral medications. Usually the hospitalization ends when the patient realizes that he or she will be no worse off at home. This is a good time to broach end of life issues. I rarely have patients in Stage IV for more than two years. A pre-terminal sign is when the patient consistently refuses to perform spirometry during an office visit. These patients rarely survive more than two months.

The above approach has served me well for many years. It is not intended as a standard or guideline for COPD. I hope that others will benefit from my observations.

Author Affliciation : Department of Internal Medicine, TTUHSC Author Contact Information: gilbert.berdine@ttuhsc.edu DOI: 10.12746/swrccc2013.0102.024 